\title{
Recommendations for Obesity Management from Singapore
}

\author{
Joan Khoo', Soo-Kiang Eng'2, Chek-Siang Foo² \\ ${ }^{1}$ Department of Endocrinology, Changi General Hospital, Singapore \\ ${ }^{2}$ National Health Group Polyclinics, Singapore \\ ${ }^{3}$ Department of Surgery, Changi General Hospital, Singapore
}

\begin{abstract}
Obesity, defined as a condition of excessive body fat with adverse effects on health, is an increasing global problem which contributes to chronic disease burden and health care costs. The Singapore Ministry of Health (MOH) released Clinical Practice Guidelines (CPG) in 2004, aimed at providing best evidence-based recommendations for diagnosis, classification, evaluation and multidisciplinary management (diet, physical activity, medication and surgery) of overweight and obesity in adults and the pediatric population. This article summarizes the guidelines, discusses their utility in Singapore and strategies for increasing their use, and briefly outlines updates in management since the CPG's publication. A revised CPG is urgently needed in view of the increasing prevalence of overweight and obesity in Singapore and the numerous advances in the management of overweight and obesity.
\end{abstract}

Keywords: obesity, weight management, clinical practice guidelines, Asia, Singapore

\section{Introduction}

Obesity is defined as a condition of excessive fat accumulation in the body, to the extent that health and well-being are adversely affected. ${ }^{1}$ It is a risk factor for numerous diseases including type 2 diabetes, coronary artery disease, infertility and cancer, and is an increasingly important global health problem. In Singapore, the prevalence of obesity [defined as body mass index (BMI) $\geq$ $30 \mathrm{~kg} / \mathrm{m}^{2}$ ] has risen from $6.9 \%$ in 2004 to $10.8 \%$ in 2010 , while the prevalence of overweight (BMI 25.0-29.9 kg/m²) was approximately $25 \% .2,3$ As expenditure on obesity in developed countries has been estimated to be up to $7 \%$ of total health costs due to associated chronic disease complications, treatment of obesity and its primary comorbidities are estimated to cost the Singapore health care system approximately $\$ 260$ million per year, not including the indirect costs of loss of productivity and absenteeism. ${ }^{4,5}$ In view of rising health care expenditure over the last three years, and the approximately 1.5 million adults in the growing Singapore population who are overweight and obese, the reduction in health costs and disability-adjusted life years, and improvement in health and quality of life, are likely to outweigh the costs of implementing government policies and campaigns to reduce overweight and obesity. ${ }^{2,3,5}$ The Singapore $\mathrm{MOH}$ CPG was released in 2004, with the intent of providing best evidence-based recommendations for diagnosis, classification, evaluation and multidisciplinary management of overweight and obesity, including the growing problem of obesity in children and adolescents. ${ }^{4}$ This article aims to provide a brief synopsis of the guidelines, including aspects which are relevant to

ISSN 0857-1074

Copyright $(2011$ by the JAFES

Received September 5, 2011. Accepted October 5, 2011.
Singapore; and discuss the utility of the recommendations for management of overweight and obesity, particularly in primary care. We will also outline updates in management published after the release of the guidelines.

\section{Summary of Methodology of CPG Development}

The stated aims of the Singapore MOH CPG for Obesity were to provide a framework to assist health care professionals in management of overweight and obesity, review available management options, and aid primary care physicians in referrals to specialists for resistant cases. ${ }^{4}$ The CPG was developed by a multidisciplinary workgroup consisting of experts in endocrinology (including a paediatric endocrinologist), psychiatry, sports medicine, bariatric surgery, and primary care; as well as representatives from dietetics, psychology, physiotherapy and the Health Promotion Board (HPB) of Singapore. The workgroup formulated these guidelines by reviewing published international guidelines and current evidence available in the research literature, and considering the characteristics of the Singaporean population. The guidelines were drawn up based on the best available evidence for management of overweight and obesity at the time of development in 2004. ${ }^{4}$ The CPG has since been withdrawn pending revision.

\section{Summary of Recommendations}

\section{Diagnosis of Obesity}

The CPG recommends the use of BMI to define obesity. Current World Health Organization (WHO) and

Corresponding author: Dr. Joan Khoo

Changi General Hospital

2 Simei Street 3 Singapore 529889

Fax number: +6567816202

Telephone number: +6568503807

E-mail address: joan_khoo@cgh.com.sg 
international guidelines recommend BMI cut-offs of 25 $\mathrm{kg} / \mathrm{m}^{2}$ and $30 \mathrm{~kg} / \mathrm{m}^{2}$ to define overweight and obesity, respectively (Table 1). ${ }^{1}$ However, the relationship between BMI and metabolic risk varies among different ethnic groups and populations. ${ }^{6-10}$ Ethnic-specific differences in body size are present in multi-racial Singapore, with overweight and obesity being more prevalent in Malay and Indian adults aged 18-69 years, compared to Chinese. ${ }^{4}$ A WHO expert consultation panel review of scientific evidence from various Asian countries including Singapore concluded that Asians, particularly individuals of South Asian (Indian) origin, have a higher percentage of body fat and risk of cardiovascular disease at this BMI cutoff than Caucasian people of the same sex and age. The panel recommended BMI values of $23 \mathrm{~kg} / \mathrm{m}^{2}$ and 27.5 $\mathrm{kg} / \mathrm{m}^{2}$ as cut-off points for public health action in Asians, based on body fat equivalence and co-morbid disease risk (Table 1). ${ }^{1,8}$ It is important that BMI cut-offs for Singapore take into account the differences between Chinese, Malays and Indians in body fat percentage and cardiovascular risk for any given BMI., ${ }^{7}$

\begin{tabular}{|c|c|c|}
\hline $\begin{array}{c}\text { Cardiovascular } \\
\text { disease risk }\end{array}$ & $\begin{array}{c}\text { Asian BMI cut-off } \\
\text { points for action } \\
\left(\mathrm{kg} / \mathrm{m}^{2}\right)\end{array}$ & $\begin{array}{l}\text { Current WHO BMI } \\
\text { cut-off points } \\
\left(\mathrm{kg} / \mathrm{m}^{2}\right)\end{array}$ \\
\hline & $<18.5$ & $<18.5$ \\
\hline Low & 18.5 to 22.9 & 18.5 to 24.9 \\
\hline Moderate & 23.0 to 27.4 & 25.0 to 29.9 \\
\hline High & 27.5 to 32.4 & 30.0 to 34.9 \\
\hline Very High & $\begin{array}{l}32.5 \text { to } 37.4 \\
\quad \geq 37.5\end{array}$ & $\begin{array}{c}35.0 \text { to } 39.9 \\
\geq 40.0\end{array}$ \\
\hline
\end{tabular}

Waist circumference (WC), measured at the mid-point between the lower costal margin and iliac crest in a standing individual with relaxed abdominal muscles, is the most practical clinical measurement for assessing the severity of abdominal obesity. Abdominal obesity is a major risk factor for development of the metabolic syndrome, type 2 diabetes mellitus and cardiovascular disease. International guidelines recommend cut-offs of $102 \mathrm{~cm}$ (male) and $88 \mathrm{~cm}$ (female) to define excess cardiovascular and metabolic risk. Based on the WHO expert consultation and data on co-morbid disease risk in the Asia-Pacific Region, the CPG recommended lower WC cut-offs of $90 \mathrm{~cm}$ (male) and $80 \mathrm{~cm}$ (female) for defining abdominal obesity in Asians.110 Ethnic- and genderspecific cut-offs are also recommended for use in the diagnosis of abdominal obesity in a 2009 consensus statement on definition of the metabolic syndrome from the International Diabetes Federation, National Heart, Lung, and Blood Institute, American Heart Association, World Heart Federation, International Atherosclerosis Society and the International Association for the Study of Obesity. ${ }^{11}$

A variety of methods may be used for body composition analysis, including skinfold thickness, bioelectrical impedance (BIA), isotope dilution techniques (such as deuterium oxide dilution), densitometry (using underwater weighing or air plethysmography), dual energy x-ray absorptiometry and 4-compartment estimation. ${ }^{4}$ However, skinfold measurement and BIA are operator- or device-dependent indirect measurements of percentage body fat; while the other methods are too expensive, time-consuming and complicated for routine clinical use.

\section{Weight Management}

Overweight and Obese Adults. The CPG recommends targeting modest weight loss in overweight and obese adults, e.g. $10 \%$ body weight over 6 months. This results in a reduction in morbidity, and is more realistic than aiming for ideal body weight. Recommendations for management focus on multidisciplinary strategies for lifestyle modification, which combine caloric restriction with increased physical activity. An algorithm for the management of obesity is provided in the CPG, with antiobesity medication and bariatric surgery as necessary (Figure 1). Patients should be evaluated for secondary causes of obesity, such as medications (including "traditional" medicine which contain corticosteroids, antipsychotics and antidepressants), and genetic or endocrine disorders (Cushing's syndrome, hypothyroidism). Evaluation for comorbidities (e.g. hypertension, diabetes mellitus, dyslipidemia, cardiovascular disease, sleep apnea, osteoarthritis) is also recommended. Investigations include thyroid function tests; screening for Cushing's syndrome; and detecting comorbidities, particularly for associated cardiovascular risk factors (e.g. electrocardiogram, fasting lipids and glucose). These should be managed according to bestpractice guidelines. The motivation for weight loss and the presence of depression and binge eating disorders must be evaluated, with subsequent behavioral therapy and psychiatric treatment when warranted. The Singapore Chinese Health Study recently found that smoking increases mortality risk in adults with $\mathrm{BMI} \geq 27.5 \mathrm{~kg} / \mathrm{m}^{2} .^{12}$ Smoking cessation efforts should be intensified in obese individuals.

Diet and increased physical activity are the first-line weight-loss strategies for all. Medications serve as adjunctive treatment for the obese individual, or the overweight patient with comorbidities. Very-low calorie diets (VLCD) and low-calorie diets (LCD) may also be used in this group. Bariatric surgery is recommended when lifestyle and pharmacological measures have failed in the severely obese with or without comorbidities (Figure 1). These measures will be discussed in greater detail below.

Diet Modification. Reduction in daily caloric intake by at least approximately 500 kilocalories will produce at least $0.5 \mathrm{~kg}$ per week weight loss. ${ }^{13}$ This may be achieved by a variety of diets with different macronutrient composition. Balanced nutrition reduction diets moderately restricted in total fat, moderate in carbohydrates (mainly complex 
carbohydrates), and moderate in protein result in loss of body weight and body fat, as long as total caloric intake is reduced. ${ }^{13,14}$ Weight loss appears to be better associated with reduced caloric intake and prolonged diet duration, rather than the macronutrient content per se. This statement is supported by a large two-year study of diets containing different percentages of carbohydrate, fat and protein, but providing the same amount of caloric restriction. The results showed that weight loss and regain was similar in all four diets studied ${ }^{14}$. The guidelines recommend that meals should not be skipped as a weight control method, and should be adequately sized so that snacks are not needed.

Commercially available meal replacements (e.g. Optifast ${ }^{\circledR}$ and Cambridge Weight Plan $^{\circledR}$ ) are effective as part of LCD and VLCD for up to 6 months for weight loss; however, sustained modification of food intake is necessary to maintain weight loss. ${ }^{15}$ Very low calorie diets may be considered in patients with BMI $\geq 30 \mathrm{~kg} / \mathrm{m}^{2}$ (commensurate Asian cut-point $27.5 \mathrm{~kg} / \mathrm{m}^{2}$ ) who have failed more conservative weight loss attempts, or those in whom rapid weight loss is a medical necessity (e.g. before bariatric surgery). They should be avoided in children; the elderly; pregnant and breastfeeding women; and patients with psychiatric problems. ${ }^{4}$ In addition to lists outlining the caloric content for meat, fish and commercially sold snacks, the CPG also includes data on the serving sizes, calorie content and fat content for food items which are commonly eaten in Singapore (e.g. chicken rice, satay, fish head curry and Asian desserts). Compliance with dietary guidelines may be limited by the high calorie and fat content of many readily available Asian foods, the cost of commercial meal replacements, and the difficulty of adhering to low-calorie diets for a prolonged period. ${ }^{4}$

Physical Activity. Moderate-intensity physical activity for 30 minutes, 3 to 5 days per week has been found to reduce cardiovascular disease and overall mortality, but has been unable to induce significant weight loss without caloric restriction. ${ }^{16,17}$ The CPG recommends starting low- to moderate-intensity physical activity for 30 to 45 minutes, 3 to 5 days per week (150 $\mathrm{min} /$ week); then gradually increasing the intensity, duration and frequency to 45 to 60 minutes on most days or every day (200-300 minutes/week) to prevent weight gain, based on the 2001 American College of Medicine (ACSM) Position Stand on exercise-based weight-loss strategies in adults. ${ }^{18}$ The 2009 update of the ACSM statement recommends further increase in physical activity for prevention of weight gain (150 to 250 minutes/week), for weight loss (> 250 minutes/week), and for prevention of weight regain (> 250 minutes/week), based on the findings of the United States National Weight Control Registry. ${ }^{19}$ However, the high levels of exercise required to achieve weight loss due to energy deficit similar to that produced by caloric restriction will be difficult for overweight or obese adults to achieve and sustain. ${ }^{16,17}$ For adults who are not able or willing to exercise continuously, the Singapore CPG

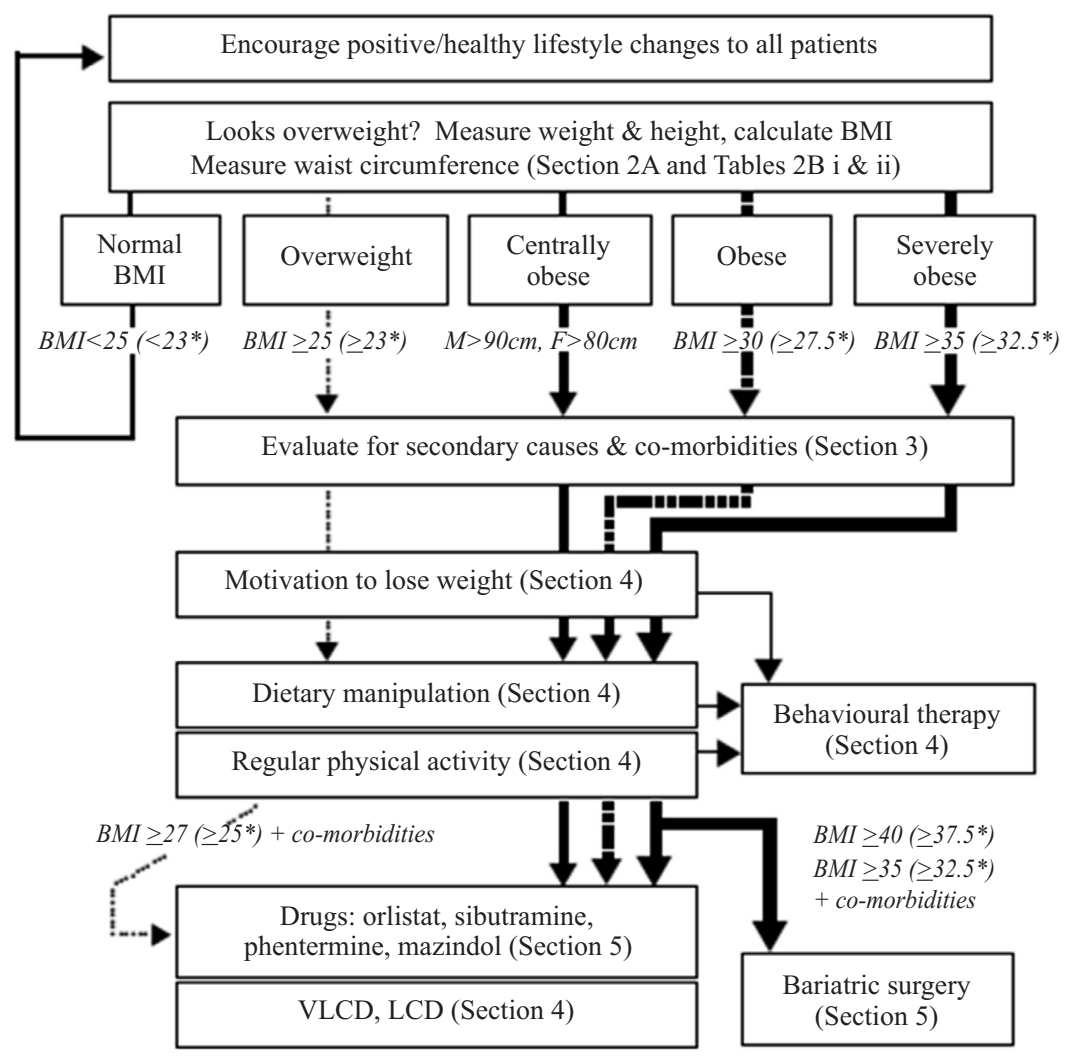

* Proposed commensurate Asian BMI cut-points for action (in $\left.\mathrm{kg} / \mathrm{m}^{2}\right)$, currently under review.

Figure 1. Algorithm for weight management from the Singapore $\mathrm{MOH} \mathrm{CPG}{ }^{4}$ 
recommends accumulation of 30 to 40 minutes of physical activity in the form of intermittent sessions lasting 10 to 15 minutes. Cumulatively, these are not superior to a continuous session of the same duration for weight loss, but do improve cardiorespiratory fitness and reduce coronary risk, and may also improve compliance. ${ }^{17}$ The CPG also provides information on caloric expenditure per 30 minutes of gym activities, sports, occupational activities and housework, which are useful for comparison with the food charts.

Behavior Modification. The CPG emphasizes the importance of increasing patient motivation for weight loss. Methods include improving understanding of obesity and associated risks for chronic disease; exploring readiness for sustained lifestyle modification; and developing strategies to address time availability, social support framework and financial status. Weight loss programs incorporating cognitive-behavioral therapy (CBT) are helpful in achieving weight loss and weight maintenance in up to $10 \%$ for one to five years of followup. ${ }^{17,20}$ Recommended CBT techniques include motivational interviewing (recognition of motives for and barriers to weight loss to facilitate behavior change), goal setting and plans of action, self-monitoring of eating habits and activity, cognitive restructuring (identifying and controlling situations which trigger unhealthy eating), stimulus control, relapse prevention and stress management. ${ }^{4,20}$ As weight regain is associated with falling-off from behavioral strategies, the CPG recommends that subjects continue with up to 12 months of a program combining behavior therapy, diet and exercise after the initial period of weight loss. However, compliance with CBT may be limited by availability and cost of referral to a psychologist; lack of time for counselling sessions; and reluctance on the part of patients, who perceive obesity to be a purely physical problem.

Anti-obesity Drugs. Pharmacological therapy is indicated for patients with BMI of at least $30 \mathrm{~kg} / \mathrm{m}^{2}$, or with BMI of $27-29.9 \mathrm{~kg} / \mathrm{m}^{2}$ in the presence of comorbidities or complications of obesity (hypertension, type 2 diabetes, hyperlipidemia, coronary artery disease and sleep apnea) who fail to lose weight with therapeutic lifestyle modification. ${ }^{4,21}$ Equivalent BMI cut-offs for Asians are 27.5 and $25-27.4 \mathrm{~kg} / \mathrm{m}^{2}$, respectively. ${ }^{4}$ At the time of publication of the CPG, sibutramine (appetite suppressant with sympathomimetic activity) and orlistat (inhibitor of digestion and absorption of ingested fat) had been approved for long-term management of obesity. In 2010, sibutramine was withdrawn from the market in North America, Europe, Australia and several Asian countries including Singapore, due to its association with increased risk of cardiovascular events in overweight and obese patients in the Sibutramine Cardiovascular Outcome Trial. ${ }^{22}$ Orlistat produces weight loss of $1.3-4.8 \%$ at a dose of $120 \mathrm{mg}$ TID at 1, 2 and 4 years, but its use is limited by gastrointestinal effects such as abdominal discomfort, bloatedness, stool leakage and steatorrhoea. ${ }^{21}$ In 2010, the U.S. Food and Drug Administration (FDA) approved a revised label for orlistat to include new safety information about cases of severe liver injury reported rarely with its use. $^{23}$

The CPG recommends the sympathomimetic appetite suppressant phentermine for short term (6 to 12 months) weight loss. Metformin is the drug of choice for weight loss in obese diabetic patients, but there is currently limited evidence on its efficacy in people without diabetes. ${ }^{24}$ The CPG also notes that the anti-epileptic agents topiramate and zonisamide are effective for inducing 5 to $6 \mathrm{~kg}$ of short-term weight loss. At the time of publication, there were limited available data on the safety and effectiveness of combining other anti-obesity drugs. At the time of writing of this article, orlistat is the only anti-obesity agent with long-term safety data approved for use in Singapore. The glucagon-like peptide-1 (GLP-1) analogues exenatide and liraglutide induced up to $7 \%$ weight loss when administered to obese type 2 diabetic and non-diabetic adults, with higher doses of liraglutide inducing significantly more weight loss (6 to $7 \mathrm{~kg}$ ) compared to orlistat $(4 \mathrm{~kg})$ in non-diabetic obese individuals. ${ }^{25-28}$ However, the use of these medications is limited by gastrointestinal side effects, and they are not currently approved for weight loss in people without diabetes..$^{25-28}$

The CPG acknowledges that pharmacological therapy is most effective when combined with diet, physical activity and behavior modification. The paucity of available antiobesity medications which are safe and effective for longterm use emphasizes the importance of caloric restriction and increased physical activity as the mainstays of weight management.

Bariatric Surgery. Bariatric surgery is defined as gastrointestinal surgery to help severely obese patients lose weight. It is more effective (16 to $35 \%$ weight loss in 2 years) than medical management (5 to $8 \%$ ) for weight loss and maintenance. ${ }^{29}$ In accordance with the U.S. National Institutes of Health (NIH) guidelines, the CPG recommended surgery for adults aged 18 to 55 with $\mathrm{BMI} \geq$ $40 \mathrm{~kg} / \mathrm{m}^{2}$ without comorbidities or $35 \mathrm{~kg} / \mathrm{m}^{2}$ with comorbidities (Asian equivalent cut-offs 37.5 and 32.5, respectively), who fail to lose weight by non-surgical methods. In the light of good results for weight loss; improvement of type 2 diabetes, the metabolic syndrome and other obesity-related comorbidities; and costeffectiveness of surgery in patients with BMI 30 to 35 $\mathrm{kg} / \mathrm{m}^{2}$; the Asian Consensus Meeting on Metabolic Surgery (ACMOMS) recommended that the BMI cut-offs be lowered to 35 and $32 \mathrm{~kg} / \mathrm{m}^{2}$, respectively; and that surgery be considered for Asian adults with BMI $\geq 30 \mathrm{~kg} / \mathrm{m}^{2}$ with central obesity (WC $>90 \mathrm{~cm}$ in males and $>80 \mathrm{~cm}$ in females) and at least two features of metabolic syndrome 
(raised triglycerides, low HDL cholesterol, hypertension, high fasting plasma glucose). ${ }^{30}$ Bariatric surgery has been performed safely and effectively in adolescents and in patients above the age of 65 in centers with high patient volumes and experienced surgeons. ${ }^{29}$

A comparative summary of the efficacy, complications and mortality of surgical procedures, such as gastric banding and gastric bypass, are available in the CPG. ${ }^{4}$ Laparoscopic sleeve gastrectomy, which was not discussed in the CPG, has become more popular in clinical practice in Singapore. Laparoscopic procedures are associated with lower morbidity and mortality, near- $100 \%$ resolution of diabetes, and improvement of other comorbidities using various techniques, such as sleeve gastrectomy, gastric banding and Roux-en-Y gastric bypass. ${ }^{31-33}$

The CPG emphasizes the importance of appropriate patient selection, thorough pre-operative evaluation, and the need for multidisciplinary management involving the physician, dietician and psychologist. Surgery entails significant technical issues, complications, and cost; necessitates extensive pre- and peri-operative preparation; and requires lifelong post-operative lifestyle modifications and follow-up for complications. Evidence from longer (more than 10 years duration) post-surgical data illustrating the improvement of mortality and comorbidities in larger populations (such as in the Swedish Obese Subjects Study), and further developments in surgical techniques, appear to support a greater role for bariatric surgery in the management of obesity in the future. ${ }^{35}$

\section{Weight Maintenance}

Obesity is a chronic condition requiring lifelong management. Clinical trials have shown that most weight is regained within five years. ${ }^{13,17}$ Based on data from U.S. National Weight Control Registry, the CPG recommends that behavioral strategies which may enhance successful long-term weight maintenance include eating a calorierestricted (approximately $1400 \mathrm{kcal} /$ day), low- to moderate-fat $(25 \%$ of total caloric intake/day) diet; frequent self-monitoring of body weight; recording food intake and physical activity; maintaining high levels of regular physical activity; and eating breakfast regularly. ${ }^{17}$ Epidemiologic, cross-sectional, and prospective correlation studies suggest an essential role for physical activity in weight-loss maintenance, and post hoc analysis of prospective trials shows a clear dose-response relationship between physical activity and weight maintenance. ${ }^{16}$ Bariatric surgery appears to be associated with greater maintenance of weight loss than medical interventions. ${ }^{29}$

\section{Obesity in Children and Adolescents}

The WHO defines childhood overweight as BMI at or above the 85th percentile and lower than the 95th percentile for children of the same age and sex, and obesity as a BMI at or above the 95th percentile, based on growth charts from the US Centers for Disease Control (CDC). ${ }^{4}$ However, the CDC charts use data derived from the U.S. population in the 1970's and are not applicable to our local setting. A recent international anthropometric survey defined the BMI percentile curves for Singapore children and teenagers up to 20 years of age, matched to adult cut-offs for overweight and obesity at 25 and 30 $\mathrm{kg} / \mathrm{m}^{2}$, respectively, positioned at age 18 years. ${ }^{35}$ The CPG recommends that either the WHO criteria or the adultmatched cut-offs may be used as thresholds for evaluation and weight management. The lack of integration of the WHO definitions with Singapore cut-offs poses difficulties in policy-making and decisions on therapeutic intervention. Moreover, the BMI percentiles corresponding to BMI 23 and $27.5 \mathrm{~kg} / \mathrm{m}^{2}$, as in the adult population, may be more appropriate for defining risk due to overweight and obesity, respectively, in Singaporean children. BMI cut-offs to define underweight in children and adolescents (based on equivalents to $\mathrm{BMI}<17 \mathrm{~kg} / \mathrm{m}^{2}$ in adults) have also been obtained for the Singapore population, and are useful for pediatric and adolescent weight management in conjunction with the corresponding definitions of overweight and obesity. ${ }^{36}$

The prevalence of obesity in Singapore children aged 6 to 16 years, based on the WHO definition, was approximately $15 \% .^{37}$ This is a disturbing statistic, as childhood obesity is an important predictor of adult obesity, and associated chronic diseases will contribute to premature morbidity and mortality. Moreover, obesity has a significant negative impact on the adolescent's healthrelated quality of life in both the physical and mental domains. ${ }^{38}$ The primary goal of childhood and adolescent obesity interventions is to adopt and maintain healthy lifestyle behaviors which still allow growth in height to continue, so that height eventually becomes appropriate for weight, or the BMI percentile becomes reduced. ${ }^{39}$ As in adults, the CPG recommends that diet modification and increased physical activity should be the mainstays of weight management in children and adolescents. Important differences for children include the use of less restrictive nutritional plans, rather than diets consisting of drastically altered nutrient composition, VLCD or lowprotein diets; and age- and weight-appropriate activities with decreased time spent on sedentary pursuits, with appropriate parental involvement. Multidisciplinary behavior-treatment programs utilizing techniques such as education, target-setting, teaching skills for weight loss, self-monitoring, stimulus control and reinforcement, have shown consistent success in weight loss..$^{39,40}$ When the CPG was developed, there was no available data on the longterm efficacy and safety of anti-obesity medications in the pediatric population. A recent meta-analysis of studies on children and adolescents found that sibutramine produced clinically meaningful reductions in BMI and improvements in cardiometabolic risk, while orlistat reduced BMI but had a high prevalence of gastrointestinal 
adverse effects. ${ }^{41}$ Although the CPG did not recommend bariatric surgery for children below 18 years due to previous paucity of data on safety and concerns about effects on growth, recent guidelines have suggested surgery for adolescents with BMI above $50 \mathrm{~kg} / \mathrm{m}^{2}$, or BMI above $40 \mathrm{~kg} / \mathrm{m}^{2}$ with severe comorbidities in whom lifestyle modifications and/or pharmacotherapy had failed. ${ }^{40}$ Centers with experienced surgeons have, with appropriate informed consent protocol for families, performed operations in girls above age 13 years and boys above age 15 years with obesity-related comorbidities. ${ }^{42}$

\section{Quality Indicators}

The CPG recommends the use of quality indicators to evaluate the success of weight management programs. Structure indicators include availability of resources and facilities to promote weight loss and maintenance in both the community and health-care settings; and a multidisciplinary weight management team consisting of the primary care doctor, nurse-educator, nutritionist, exercise therapist, behavioural therapist, endocrinologist with specialized training in weight management, respiratory physician, cardiologist and surgeon where necessary. Regular monitoring of performance parameters is recommended: patient education, weight, BMI, WC and blood pressure at least quarterly; and fasting glucose and lipids at least annually. Data on outcome indicators (percentage of weight loss; amount of weight regain; and reduction in $\mathrm{BP}, \mathrm{LDL}$ cholesterol and blood glucose) should be collected from weight management programs in the community and health-care providers.

\section{Engaging Physicians in the Use of the CPG}

The majority $(80 \%)$ of the CPG's stated target group of primary care practitioners provide care in approximately 2000 private clinics situated throughout Singapore. ${ }^{43}$ Referrals to psychologists, dieticians and physical trainers are not readily available in the community as part of a structured weight management program. Physicians and patients may also perceive these additional interventions to be expensive and time-consuming. There are no published data on the rate of implementation of the CPG in the community, or its association with weight loss and improvement in cardiovascular risk factors and obesityrelated comorbidities. As such, there is a pressing need to evaluate the effectiveness of the CPG (and subsequent revisions), and to increase engagement of primary care physicians in the adoption of these recommendations. Cost-effective tools, such as postal surveys with incentives for participation, can be developed to monitor CPG usage, obtain feedback on difficulties in compliance, and identify barriers and needs in obesity management in the community. ${ }^{44}$ Follow-up targeted clinical tools such as facilitated two-way referral pathways to community-based dieticians, behavioural and sports therapists; and wider access to educational materials and multimedia resources on-line and in print; are useful to bridge the gap in care between general practitioners and specialists in obesity management. ${ }^{45}$ Incremental pacing of the introduction of initiatives and guidelines will be helpful in the integration of care and resources in the primary care sector with public health and hospital-based facilities. Involvement of patients will also be facilitated by availability of on-line resources for weight management, such as websites and smartphone applications. Indeed, interactive technologybased interventions have been shown to be superior to self-directed weight control in limiting regain at 24 months after completion of a weight loss program. ${ }^{46}$

\section{Conclusion}

The Singapore $\mathrm{MOH}$ Clinical Practice Guidelines for Obesity (2004) emphasize the importance of multidisciplinary involvement in weight management, and individualization of management according to patient needs and available diagnostic and treatment options. Its strengths include contributions from relevant allied medical disciplines and community health care experts in addition to medical professionals, the use of the best evidence available at the time of its inception, and inclusion of recommendations and information which are uniquely useful for the local population. Nevertheless, the prevalence of obesity increased after the CPG was released, underscoring the need for measures to improve uptake of the recommendations and acceptability and availability of health care resources. An updated CPG is urgently needed in view of the rapid and numerous advances in knowledge and tools for the management of overweight and obesity.

\section{References}

1. WHO Expert Consultation. Appropriate Body-Mass Index for Asian populations and its implications for policy and intervention strategies. Lancet. 2004;363:157-63.

2. National Health Survey 2004. Epidemiology and Disease Control Department, Ministry of Health, Singapore.

3. National Health Survey 2010. Epidemiology and Disease Control Department, Ministry of Health, Singapore.

4. Clinical Practice Guidelines 5/2004. Obesity. Ministry of Health, Singapore. World Health Organization.

5. Ministry of Health Singapore: Government Health Expenditure. http://www.moh.gov.sg/mohcorp/statistics.aspx?id=5972.

6. Misra A, Khurana L. Obesity and the metabolic syndrome in developing countries. J Clin Endocrinol Metab. 2008;93:S9-30.

7. Deurenberg-Yap M, Chew SK, Lin VF et al. Relationships between indices of obesity and its comorbidities in multi-ethnic Singapore. Int J Obes Relat Metab Disord. 2001;25:1554-62.

8. Deurenberg P, Deurenberg-Yap M, Guricci S. Asians are different from Caucasians and from each other in their Body Mass Index/body fat percent relationship. Obes Rev. 2002;3:141-6.

9. Deurenberg-Yap M, Chew SK, Deurenberg P. Elevated body fat percentage and cardiovascular risks at low Body Mass Index levels among Singaporean Chinese, Malays and Indians. Obes Rev. 2002;3:209-15.

10. The Asia-Pacific perspective: Redefining obesity and its treatment. Melbourne: WHO Western Pacific Regional Office, IASO, IOTF, 2000. http://www.obesityasiapacific.com/pdf/obesity.pdf.

11. Alberti KG, Eckel RH, Grundy SM et al. Harmonizing the metabolic syndrome: A joint interim statement of the International Diabetes Federation Task Force on Epidemiology and Prevention; National Heart, Lung, and Blood Institute; American Heart Association; World Heart Federation; International Atherosclerosis Society; and 
International Association for the Study of Obesity. Circulation. 2009;120:1640-5.

12. Odegaard AO, Pereira MA, Koh W-P et al. BMI, all-cause and causespecific mortality in Chinese Singaporean men and women: The Singapore Chinese Health Study. PLoS One. 2010;5(11):e14000.

13. Franz MJ, VanWormer JJ, Crain AL et al. Weight-loss outcomes: A systematic review and meta-analysis of weight-loss clinical trials with a minimum 1-year follow-up. J Am Diet Assoc. 2007;107:1755-67.

14. Sacks FM, Bray GA, Carey VJ et al. Comparison of weight-loss diets with different compositions of fat, protein, and carbohydrates. N Engl J Med. 2009;360:859-73.

15. Heymsfield SB, van Mierlo CAJ, van der Knaap HCM, Heo M, Frier HI. Weight management using a meal replacement strategy: Meta and pooling analysis from six studies. International Journal of Obesity. 2003;27:537-49.

16. Catenacci VA, Wyatt HR. The role of physical activity in producing and maintaining weight loss. Nat Clin Pract Endocrinol Metab. 2007;3:518-52.

17. Wing RR, Phelan S. Long-term weight loss maintenance. Am J Clin Nutr. 2005;82:222S-5S

18. Jakicic JM, Clark K, Coleman E et al. American College of Sports Medicine position stand. Appropriate intervention strategies for weight loss and prevention of weight regain for adults. Med Sci Sports Exerc. 2001;33:2145-56.

19. Donnelly JE, Blair SN, Jakicic JM et al. American College of Sports Medicine position stand. Appropriate physical activity intervention strategies for weight loss and prevention of weight regain for adults. Med Sci Sports Exerc. 2009;41:459-71

20. Levy RL, Finch EA, Crowell MD, Talley NJ, Jeffery RW. Behavioral intervention for the treatment of obesity: Strategies and effectiveness data. Am J Gastroenterol. 2007;102:2314-21.

21. Li Z, Maglione M, Tu W et al. Meta-analysis: Pharmacologic treatment of obesity. Ann Intern Med. 2005;142:532-46.

22. James WP, Caterson ID, Coutinho $\mathrm{W}$ et al. Effect of sibutramine on cardiovascular outcomes in overweight and obese subjects. N Engl J Med. 2010;363:905-17

23. U.S. Food and Drug Administration. FDA Drug Safety Communication: Completed safety review of Xenical/Alli (orlistat) and severe liver injury. May 26, 2010.

24. Desilets AR, Dharkal-Karki S, Dunican KC. Role of metformin for weight management in patients without type 2 diabetes. Ann Pharmacother. 2008:42:817-26

25. Ratner RE, Maggs D, Nielsen LL et al. Long-term effects of exenatide therapy over 82 weeks on glycemic control and weight in over-weight metformin-treated patients with type 2 diabetes mellitus. Diab Obes Metab. 2006;8:419-28

26. Buse JB, Rosenstock J, Sesti G et al for the LEAD-6 study group. Liraglutide once a day versus exenatide twice a day for type 2 diabetes: A 26-week randomised, parallel-group, multinational, openlabel trial (LEAD-6).Lancet. 2009;374:39-47.

27. Rosenstock J, Klaff LJ, Schwartz S et al. Effects of exenatide and lifestyle modification on body weight and glucose tolerance in obese subjects with and without pre-diabetes. Diabetes Care. 2010;33:1173-5

28. Astrup A, Rössner S, Van Gaal L et al. Effects of liraglutide in the treatment of obesity: A randomised, double-blind, placebo-controlled study. Lancet. 2009;374:1606-16.
29. Buchwald $\mathrm{H}$, Avidor $\mathrm{Y}$, Braunwald $\mathrm{E}$ et al. Bariatric surgery: A systematic review and meta-analysis. JAMA. 2004;292:1724-37.

30. Lakdawala M, Bhasker A. Asian Consensus Meeting on Metabolic Surgery (ACMOMS). Recommendations for the use of bariatric and gastrointestinal metabolic surgery for treatment of obesity and type 2 diabetes mellitus in the Asian population. Obes Surg. 2010;20:929-36.

31. Han SM, Kim WW, Hyun Oh J. Results of laparoscopic sleeve gastrectomy (LSG) at 1 year in morbidly obese Korean patients. Obes Surg. 2005;15:1469-75.

32. O'Brien P, Dixon JB, Laurie $\mathrm{C}$ et al. Treatment of mild to moderate obesity with laparoscopic adjustable gastric banding or an intensive medical program: A randomized trial. Ann Intern Med. 2006;144:62533.

33. Higa $\mathrm{K}, \mathrm{Ho} \mathrm{T}$, Terecero $\mathrm{F}$ et al. Laparoscopic Roux-en- $\mathrm{Y}$ gastric bypass: 10-year follow-up. Surg Obes Relat Dis. 2011;7:516-25.

34. Sjöström L, Narbro K, Sjöström CD et al. Effects of bariatric surgery on mortality in Swedish obese subjects. N Engl J Med. 2007;357:74152.

35. Cole TJ, Bellizzi MC, Flegal KM et al. Establishing a standard definition for child overweight and obesity worldwide: International survey. BMJ. 2000;320:1240-3.

36. Cole TJ, Flegal KM, Nicholls DA, Jackson AA. Body Mass Index cutoffs to define thinness in children and adolescents: international survey. BMJ. 2007;335:194-201.

37. Annual Report. Singapore: School Health Services, Singapore. 2000

38. Wee HL, Wu Y, Thumboo J, Lee J, Tai ES. Association of Body Mass Index with Short-Form 36 physical and mental component summary scores in a multiethnic Asian population. Int J Obes (Lond). 2010;34:1034-43.

39. Kamath CC, Vickers KS, Ehrlich A et al. Behavioral interventions to prevent childhood obesity: A systematic review and metaanalyses of randomized trials. J Clin Endocrinol Metab. 2008;93:4606-15.

40. August GP, Caprio C, Fennoy I et al. Prevention and treatment of pediatric obesity: An Endocrine Society clinical practice guideline based on expert opinion. J Clin Endocrinol Metab. 2008;93:4576-99.

41. Viner RM, Hsia Y, Tomsic T, Wong ICK. Efficacy and safety of antiobesity drugs in children and adolescents: Systematic review and meta-analysis. Obes Rev. 2010;11:593-602.

42. Sinha A, Scott K. A review of adolescent obesity: Prevalence, etiology and treatment. Obes Surg. 2009;19:113-20.

43. Ministry of Health, Singapore: Healthcare Services (Primary Care) http://www.moh.gov.sg/mohcorp/hcservices.aspx?id=392. Accessed October 4, 2011.

44. Hutchinson A, McIntosh A, Cox S, Gilbert C. Towards efficient guidelines: How to monitor guideline use in primary care. Health Technol Assess. 2003;7(iii):1-97.

45. Hopkins KF, Decristofaro C, Elliott L. How can primary care providers manage pediatric obesity in the real world? J Am Acad Nurse Pract. 2011;23:278-88.

46. Svetkey LP, Stevens VJ, Brantley PJ et al. Comparison of strategies for sustaining weight loss: The weight loss maintenance randomized controlled trial. JAMA. 2008;299:1139-48.

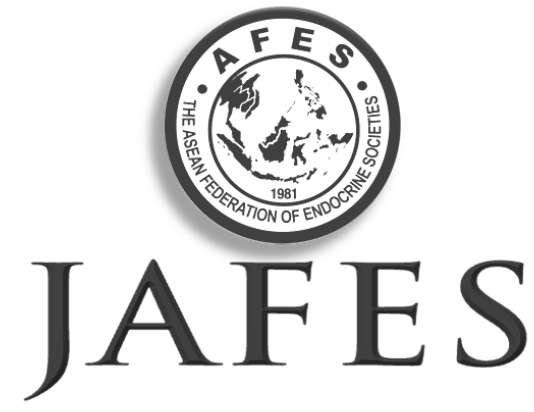

Experience the new JAFES.
Visit us at www.ASEAN-endocrinejournal.org. 